\title{
Re-engagement in HIV care following a missed visit in rural Uganda
}

\author{
Maria Sarah Nabaggala ${ }^{1 *}$, Rosalind Parkes-Ratanshi ${ }^{1,2}$, Ronnie Kasirye ${ }^{1}$, Agnes Kiragga ${ }^{1}$, Barbara Castlenuovo ${ }^{1}$, \\ lan Ochaka', Lilian Nakakawa', Diana Asiimwe Bena ${ }^{1}$ and Andrew Mujugira ${ }^{1,3}$
}

\begin{abstract}
Objective: We conducted a retrospective cohort study to assess the effect of tracking People Living with HIV (PLHIV) after missed clinic visits and factors associated with return to care in rural Uganda. We assessed retention in care among 650 HIV-infected women and men. We used univariable and multivariable generalized linear models to assess demographic and self-reported factors associated with re-engagement in HIV care.

Results: Of 381 PLHIV who ever missed a scheduled appointment, 68\% were female and most (80\%) had initiated ART. Most (70\%) of those tracked returned to care. Relative to men, women (adjusted risk ratio [ARR] 1.23; 95\% confidence interval (CI) 1.05-1.43; $p=0.009$ ) were more likely to return to care after active tracking. PLHIV who missed scheduled visits for other reasons (forgetting, adequate drug supplies, or long distance to clinic) had reduced odds of return to care (ARR $0.41 ; 95 \% \mathrm{Cl} 0.28-0.59 ; \mathrm{p}<0.001$ ). These data support close monitoring of patient retention in HIV care and active measures to re-engage those who miss an appointment. Furthermore, they highlight the need for targeted interventions to those more resistant to re-engagement such as men.
\end{abstract}

Keywords: HIV, ART, Retention, PLHIV tracking, Return to care

\section{Introduction}

Uganda is one of 15 countries that accounted for $75 \%$ of the 2.1 million new HIV infections that occurred in 2015 [1]. Uganda has scaled up coverage of antiretroviral therapy (ART), which durably suppresses plasma and genital tract viral load, decreases HIV-associated mortality, and prevents HIV transmission [2,3]. The proportion of HIV-infected adults receiving ART in Uganda increased from an estimated $37-57 \%$ in 2011 to $81 \%$ in $2016 / 2017$ [4]. Increased ART coverage was accompanied by a $51 \%$ decrease in the number of new HIV infections and a $45 \%$ reduction in HIV-related mortality between 2010 and 2016 [5].

Patient retention in HIV care is key to achieving Joint United Nations Programme on HIV/AIDS (UNAIDS) and national 90-90-90 targets: $90 \%$ of all people with HIV to be diagnosed and know their status, $90 \%$ of all

\footnotetext{
*Correspondence: snabaggala@idi.co.ug

${ }^{1}$ Infectious Diseases Institute, College of Health Sciences, Makerere University, Kampala, Uganda

Full list of author information is available at the end of the article
}

HIV-infected people to receive ART, and $90 \%$ of persons receiving ART to be virally suppressed by 2020 [6, 7]. Continuous engagement with the healthcare system by HIV infected individuals is a key challenge for HIV treatment programs [8]; losses occur at each step of the HIV care cascade [9], and most attrition occurs during the first 24 months of ART [10]. Despite the personal and public health benefits of HIV treatment, global retention in care on ART at 12 months was approximately $74 \%$ in 2016 [11]. In Uganda, attrition rates of up to $20 \%$ have been reported 12 months after ART initiation [12].

Persons not retained in care experience treatment interruptions and viral non-suppression, which increases risk of drug resistant virus, and compromises personal and population-level benefits of ART [11]. Although no uniform definition of retention in care exists, at least one visit to an HIV care provider every 6 months is typically used to allow for clinically stable and virologically suppressed PLHIV who require fewer provider interactions [13-17]. Strategies to improve retention in HIV care include peer support, targeted counselling, mobile phone tracking and home visits [13]. Targeted 
retention interventions could minimize ART interruptions and reduce HIV-related morbidity and mortality [11]. We undertook a study at a rural government hospital in North-Eastern Uganda, with specific challenges of an agro-pastoralist community which practices seminomadic livestock rearing and whose settlement patterns are determined by availability of pasture and water sources [18]. We assessed the effect of patient tracking using phone calls and/or home visits on return to care among PLHIV who missed their scheduled visits.

\section{Main text \\ Methods \\ Population and procedures}

Between January 2014 and August 2015, we conducted a retrospective cohort study of PLHIV receiving HIV care at Moroto Regional Referral Hospital (RRH). The HIV program at this facility was supported by the Civil Society Fund Regional Referral Hospitals Project [19]. We included in the study PLHIV who enrolled in the ART clinic during the study period, missed a scheduled visit, and had medical records in the Open Medical Records System $\left(\right.$ OpenMRS $\left.{ }^{\circledR}\right)$ database. Those who missed a scheduled clinic visit were contacted by telephone after 5 days (Fig. 1). The clinic had a designated counsellor to actively track persons who missed one or more visits. If the PLHIV had no telephone contact or failed to return to the clinic after several calls, a home visit was conducted by clinic staff, an expert client or a peer leader. PLHIV were tracked for 3 consecutive months after a missed scheduled visit. Those who did not return were flagged as lost to follow-up. These active tracking measures were intended to encourage PLHIV to return to active follow-up, address psychosocial issues and better understand circumstances that led to failure to turn up for the scheduled clinic visit. Data on PLHIV age, gender, ART status, tracking method, return to clinic, and reason(s) for missed visits were collected on PLHIV tracking/follow-up forms.

\section{Statistical analysis}

Return to care (the primary outcome) was defined as return to care within 3 months of being tracked. Descriptive statistics and frequency distributions were performed for continuous and categorical data, respectively. To identify factors associated with returning to care after active tracking, we categorized PLHIV who were tracked after missing an appointment by whether they did or did not return to care. A generalized linear model was used to estimate risk ratios of return to care after adjusting for underlying differences in predictor variables (age, gender, ART status, mode of contact and reason(s) for missing scheduled clinic visit). Significance was set at $\mathrm{p}<0.05$. Data were analyzed using Stata version 14 (StataCorp LLC, College Station, Texas).

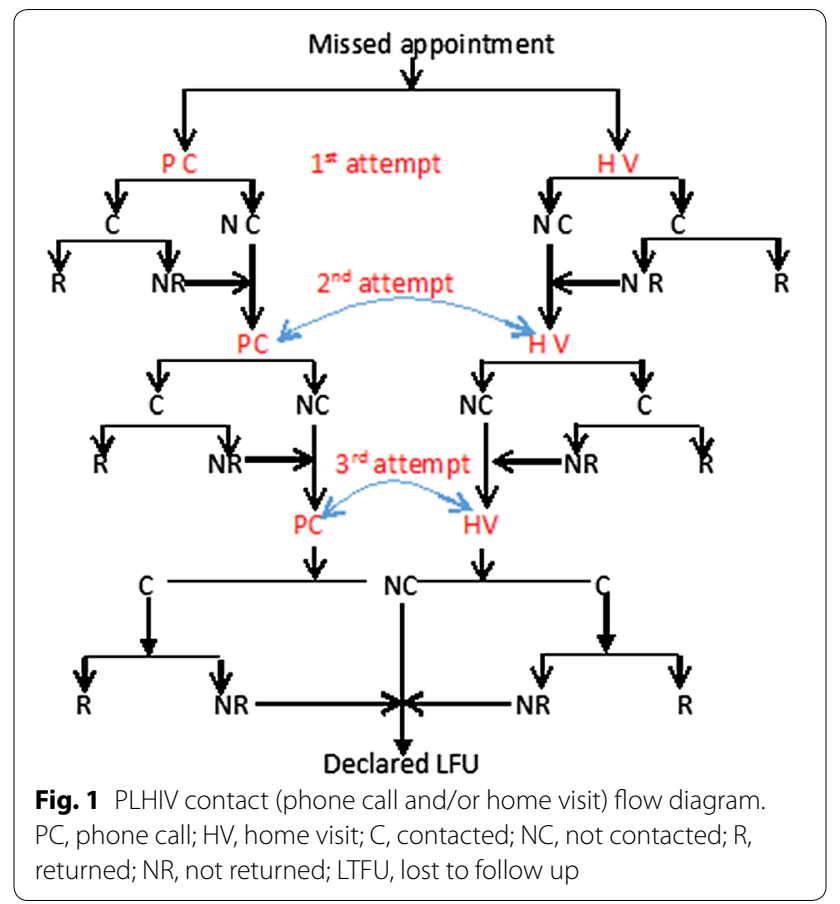

\section{Results}

Of the 650 persons in HIV care during the study period, 381 (59\%) had ever missed a scheduled clinic visit and were included in the present analysis. For these 381, the median age was 30 years [interquartile range (IQR), 23-35], 259 (68\%) were female and 306 (80\%) had initiated ART (Table 1). Overall, 598 phone calls and 472 home visits were conducted during the study period.

Seventy percent of PLHIV tracked by phone call and/or home visits returned to care. Of these, $74 \%$ were women. PLHIV were as likely to return to care after being tracked through a phone call as through home visit (73\% vs. $64 \%$; $\mathrm{p}=0.08$ ). Of the $42 \%$ who self-reported missing scheduled clinic visits because of having travelled away from home, most (67\%) were women. Forty-three percent reported socio-structural barriers to retention in care including forgetting the scheduled appointment, long distance to clinic, tight work schedules, stigma, ART side effects, having adequate supplies of drugs, and lack of food. The remaining $15 \%$ were unreachable or not available when tracked by phone and/or home visit.

In multivariable analyses, women were more likely to return to care after active tracking than men (adjusted risk ratio $[A R R] 1.23 ; 95 \%$ CI 1.05-1.43; $\mathrm{p}=0.009)$. Compared with persons who had travelled, those who were unavailable/unreachable at time of contact (ARR 0.85; 95\% CI 0.75-0.95; $\mathrm{p}=0.01$ ) and those that reported other reasons for missing their scheduled appointments e.g., long distance to clinic, stigma, still having drug supplies 
Table 1 Distribution of patient characteristics stratified by return to HIV care and predictors of return to care having missed a scheduled appointment after tracking

\begin{tabular}{|c|c|c|c|c|c|}
\hline Characteristic N (\%) or median (IQR) & $\begin{array}{l}\text { Did not return to HIV } \\
\text { care }(n=114)\end{array}$ & $\begin{array}{l}\text { Returned to HIV } \\
\text { care }(n=267)\end{array}$ & p-value & Adjusted OR (95\% Cl) & p-value \\
\hline Age in years, median (IQR) & $30(25-36)$ & $29(22-34)$ & 0.01 & $0.99(0.98-1.01)$ & 0.265 \\
\hline \multicolumn{6}{|l|}{ Gender } \\
\hline Male & $53(43.4)$ & $69(56.6)$ & $<0.001$ & Reference & \\
\hline Female & $61(23.5)$ & $198(76.5)$ & & $1.23(1.05-1.43)$ & 0.009 \\
\hline \multicolumn{6}{|l|}{ ART status } \\
\hline ART naive & $33(44.0)$ & $42(56.0)$ & 0.003 & Reference & \\
\hline On ART & $81(26.5)$ & $225(73.5)$ & & $1.16(0.96-1.39)$ & 0.119 \\
\hline \multicolumn{6}{|l|}{ Tracking method } \\
\hline Phone call & $63(26.7)$ & $173(73.3)$ & 0.08 & Reference & \\
\hline Home visit & $51(35.2)$ & $94(64.8)$ & & $0.98(0.87-1.11)$ & 0.832 \\
\hline \multicolumn{6}{|l|}{ Reason for missing appointment } \\
\hline Travelled & $51(31.5)$ & $111(68.5)$ & $<0.001$ & Reference & \\
\hline Unavailable & $38(67.9)$ & $18(32.1)$ & & $0.85(0.75-0.95)$ & 0.006 \\
\hline Other & 25 (15.3) & $138(84.7)$ & & $0.41(0.28-0.59)$ & $<0.001$ \\
\hline
\end{tabular}

or forgetting the appointment (ARR 0.41; 95\% CI 0.28$0.59 ; \mathrm{p}<0.001)$ were less likely to return to care after missing a scheduled visit. Age, ART status and tracking method were not related to return to care $(\mathrm{p}=0.27,0.12$ and 0.83 , respectively).

\section{Discussion}

In this retrospective cohort study of HIV-infected Ugandan adults, in which PLHIV who missed scheduled visits were actively followed through phone contact and/or home visits, most returned to care after being tracked. Female gender and prior receipt of ART were predictors of return to HIV care. Those who reported nontravel reasons for missed visits, or were unreachable by designated personnel, were significantly less likely to be retained in HIV care.

We found that women and PLHIV on ART were more likely to return to HIV care after missing a scheduled clinic visit. This finding is in agreement with prior studies in which gender and ART status were associated with return to care after tracking [16, 20-22]. In a systematic review of 42 studies from 12 countries, factors associated with lower retention rates included younger age, male gender, stigma, non-disclosure of HIV status, fear of drug side effects, and transport costs [23]. Other studies have found that denial of HIV status, healthy status perception, substance use, mental illness, distrust of medical providers, and side effects of HIV medications were associated with poorer retention [14, 23, 24]. Addressing these factors could increase the likelihood of staying engaged and retained in HIV care. In our study, a significantly higher proportion of men did not re-engage in care after active tracking. In East and Southern Africa, ART coverage is lower among men than women $(57 \%$ vs $72 \%$ ) [25], and men are less likely than women to adhere to HIV treatment resulting in poorer outcomes [26, 27]. Cultural constructs of masculinity and HIV stigma appear to influence health seeking behavior and compromise HIV care utilization in this setting [27]. HIV providers should utilize culturally appropriate initiatives to encourage health-enabling masculinities and support retention in care.

The proportion of PLHIV who ever missed a scheduled clinic visit in our study is similar to that reported in other studies of mobile populations [15, 16, 22]. Some PLHIV may have cycled in and out of care, given the mobile nature of the study population [9]. We observed that most PLHIV who were traced re-engaged in HIV care. Active tracing and linkage to care likely account for these findings. Monitoring retention on ART is key to estimating the proportion disengaging from care and developing targeted interventions to improve engagement in care, decreasing mortality, and minimizing adverse treatment outcomes [11]. Ancillary services such as case management, outreach, support groups, and patient navigation could further improve retention in care and facilitate attainment of national and global HIV treatment goals [28-33].

In our study, clinical, structural and psychosocial barriers to retention in care included long distance travel to clinic, tight work schedules, stigma, ART side effects, forgetting the scheduled appointment and food insecurity. This study was undertaken in Karamoja region. The region has poorer health outcomes than elsewhere 
in Uganda due to a combination of environmental, geographical, economic and cultural issues leading to conflict, food insecurity and malnutrition, high alcohol rates, gender based violence and low education rates [34]. Despite these difficulties, our findings are in striking agreement with other work from sub-Saharan Africa in which $65 \%$ reported structural barriers (transportation, distance to clinic, poverty), 33\% reported clinic-based barriers (long waiting times), and 27\% reported psychosocial barriers (social support, stigma and non-disclosure of HIV status) to retention in care [35]. These sociostructural factors are major determinants of retention in HIV care [36]. Accessing HIV care closer to home may decrease the cost and disruption of ART non-adherence. Community-based ART delivery programs, in which ART is delivered to consenting, stable participants at community drug distribution points, improve access to HIV treatment services, ART adherence and retention in care in resource-limited settings [37]. This alternative delivery model provides drug refills every $2-3$ months, decreases waiting times, reduces travel time and costs, and provides psychosocial support by expert clients, thereby reducing the impact of socio-structural barriers on retention in care [37]. Community drug distribution points are an effective alternative delivery model which should be scaled up to improve retention in care in resource-limited settings.

\section{Conclusion}

In conclusion, we found that nearly half of HIV-infected adults attending a rural HIV clinic in a region with poor health outcomes had ever missed a scheduled visit. Seven-in-ten PLHIV returned to HIV care following active tracing using home visits and phone calls. These data support active monitoring of patient retention in ART care and highlight the need for targeted interventions to engage men and others who struggle to maintain engagement in care especially in challenging environments.

\section{Limitations}

Our study has limitations. We did not collect mortality data and those who returned to care may have been more likely to be healthy. Data were collected from a public sector clinic and there were considerable missing data. We did not evaluate socio-economic variables including level of education, occupation, marital status and disclosure of HIV status because these data are not routinely collected. Finally, we did not document CD4 counts, HIV viral load, and ART regimen to objectively assess HIV treatment outcomes after return to care.
These factors may limit the generalizability of our findings. Nevertheless, we demonstrated that a substantial proportion of PLHIV re-engaged in HIV care following active tracing in a rural, resource-limited ART clinic.

\section{Abbreviations}

HIV: human immunodeficiency virus; PLHIV: people living with human immunodeficiency virus; ARR: adjusted risk ratio; Cl: confidence interval; ART : antiretroviral therapy; UNAIDS: United Nations Programme on HIV/AIDS; RRF: Regional Referral Hospital; CSF: Civil Society Fund.

\section{Authors' contributions}

$D A B$ and RPR designed the intervention. MSN and RPR designed the retrospective study. MSN and AM wrote the first draft. MSN performed the statistical analyses. RK edited and assisted in the writing of second draft. AK supervised the statistical analysis and edited the subsequent drafts of the manuscript. BC edited and assisted in the writing of the manuscript various drafts. IO collected the data and also drafted the manuscript. LN contacted the study participants and helped with the proof reading of the manuscript. All authors contributed to data collection, interpretation of the results and the writing of the manuscript, and all approved the final draft. All authors read and approved the final manuscript.

\section{Author details}

${ }^{1}$ Infectious Diseases Institute, College of Health Sciences, Makerere University, Kampala, Uganda. ${ }^{2}$ Cambridge Institute of Public Health, University of Cambridge, Cambridge, UK. ${ }^{3}$ Department of Epidemiology and Biostatistics, College of Health Sciences, Makerere University, Kampala, Uganda.

\section{Acknowledgements}

We thank study participants for their invaluable contributions. We thank the management and staff of Moroto Regional Referral Hospital for assistance with data collection.

These data were reported, in part, at the 2nd Edition UNESCO-MERCK Africa Research Summit (UNESCO-MARS), 28-29th November 2016, Addis Ababa, Ethiopia (poster abstract).

\section{Competing interests}

The authors declare that they have no competing interests.

\section{Availability of data and materials}

The dataset used and/or analyzed during the current study are available from the corresponding author on reasonable request.

\section{Consent for publication}

Not applicable.

\section{Ethics statement}

Ethical approval for this study was granted by the Joint Clinical Research Center Institutional Review Board and the Uganda National Council for Science and Technology (HS 1631). Due to the retrospective study design and strict anonymity of participants, the need for patient consent was waived by UNCST.

\section{Funding source}

This work was supported by a Civil Society Fund grant to the Infectious Diseases Institute (Grant CSF \#01-10-003). Support for manuscript writing was provided by the Fogarty International Center, National Institutes of Health (Grant \#D43 TW009771 "HIV co-infections in Uganda: TB, Cryptococcus, and Viral Hepatitis" and Grant \#K43 TW010695 [to AM]).

Role of the funding source The authors designed and executed the study, had full access to the raw data, performed all analyses, wrote the manuscript, and had final responsibility for the decision to submit for publication. The funder had no role in design, data collection, analysis, interpretation, or writing of the report. 


\section{Publisher's Note}

Springer Nature remains neutral with regard to jurisdictional claims in published maps and institutional affiliations.

Received: 31 August 2018 Accepted: 17 October 2018

Published online: 25 October 2018

\section{References}

1. WHO. Progress report 2016: prevent HIV, test and treat all: WHO support for country impact. 2016. http://www.who.int/iris/handle/10665/251713. Accessed 3 July 2018

2. Lundgren JD, Babiker AG, Gordin F, Emery S, Grund B, Sharma S, et al. Initiation of antiretroviral therapy in early asymptomatic HIV infection. N Engl J Med. 2015;373(9):795-807.

3. Cohen MS, Chen YQ, McCauley M, Gamble T, Hosseinipour MC, Kumarasamy N, et al. Antiretroviral therapy for the prevention of HIV-1 transmission. N Engl J Med. 2016:375(9):830-9.

4. WHO. Global update on HIV treatment 2013: results, impact and opportunities. 2013

5. UNAIDS. Uganda Country Factsheet. 2017 [Internet]. Available from: http://www.unaids.org/en/regionscountries/countries/uganda. Accessed 2 July 2018

6. UNAIDS. Joint United Nations Programme on HIV/AIDS. 90-90-90: an ambitious treatment target to help end the AIDS epidemic. 2014.

7. Uganda Ministry of Health. UAC. National HIV and AIDS Strategic Plan 2015/2016-2019/2020 [Internet]. Available from: http://library.health.go. ug/publications/service-delivery-diseases-control-prevention-commu nicable-diseases/hivaids/national-h-1. Accessed 23 June 2018.

8. Rachlis B, Bakoyannis G, Easterbrook P, Genberg B, Braithwaite RS, Cohen CR, Bukusi EA, Kambugu A, Bwana MB, Somi GR, Geng EH. Facility-level factors influencing retention of patients in HIV care in East Africa. PloS one. 2016 Aug 10;11(8):e0159994.

9. Kranzer K, Govindasamy D, Ford N, Johnston V, Lawn SD. Quantifying and addressing losses along the continuum of care for people living with HIV infection in sub-Saharan Africa: a systematic review. J Int AIDS Soc. 2012;15(2):17383.

10. Hassan AS, Mwaringa SM, Ndirangu KK, Sanders EJ, de Wit TF, Berkley JA. Incidence and predictors of attrition from antiretroviral care among adults in a rural HIV clinic in Coastal Kenya: a retrospective cohort study. BMC Public Health. 2015;15:478.

11. WHO. Global report on early warning indicators of HIV drug resistance: technical report 2016.

12. WHO. Retention in HIV Programmes. Defining the challenges and identifying solutions. Meeting report 13-15 September 2011 [Internet]. Available from: https://whqlibdoc.who.int/publications/2012/97892 41503686_eng.pdf. Accessed 2 Sept 2018.

13. Marks G, Gardner LI, Craw J, Crepaz N. Entry and retention in medical care among HIV-diagnosed persons: a meta-analysis. AIDS. 2010;24(17):2665-78.

14. Mugavero MJ, Amico KR, Horn T, Thompson MA. The state of engagement in HIV care in the United States: from cascade to continuum to control. Clin Infect Dis. 2013:57(8):1164-71.

15. Mugavero MJ, Davila JA, Nevin CR, Giordano TP. From access to engagement: measuring retention in outpatient HIV clinical care. AIDS Patient Care STDS. 2010;24(10):607-13.

16. Mugavero MJ, Lin HY, Willig JH, Westfall AO, Ulett KB, Routman JS, et al. Missed visits and mortality among patients establishing initial outpatient HIV treatment. Clin Infect Dis. 2009;48(2):248-56.

17. Horstmann E, Brown J, Islam F, Buck J, Agins BD. Retaining HIV-infected patients in care: where are we? Where do we go from here? Clin Infect Dis. 2010;50(5):752-61.

18. UNDP. KARAMOJA: MOROTO district, hazard, risk and vulnerability profile. 2014. http://www.ug.undp.org/content/uganda/en/home/library/crisi s_prevention_and_recovery/karamoja-moroto-district-hazard-risk-andvulnerability-profil.html.

19. Infectious Diseases Institute. Annual Report. 2012. Investing in the future:Impacting Real Lives [Internet]. Available from: https://www.
idi-makerere.com/wp-content/uploads/2017/08/IDI-Annual-Repor t-2012.pdf. Accessed 9 Sept 2018.

20. Cunningham CO, Buck J, Shaw FM, Spiegel LS, Heo M, Agins BD. Factors associated with returning to HIV care after a gap in care in New York State. Journal of acquired immune deficiency syndromes (1999). 2014;66(4):419.

21. Cabral HJ, Tobias C, Rajabiun S, Sohler N, Cunningham C, Wong M, et al. Outreach program contacts: do they increase the likelihood of engagement and retention in HIV primary care for hard-to-reach patients? AIDS Patient Care STDS. 2007;21(Suppl 1):S59-67.

22. Nakigozi G, Makumbi FE, Kigozi G, Nalugoda F, Reynolds SJ, Chang LW, et al. Barriers to utilization of HIV care services among adolescents and young adults in Rakai, Uganda: the role of economic strengthening. Global Soc Welfare. 2015;2(2):105-10.

23. Govindasamy D, Ford N, Kranzer K. Risk factors, barriers and facilitators for linkage to antiretroviral therapy care: a systematic review. AIDS. 2012;26(16):2059-67.

24. Kushel MB, Colfax G, Ragland K, Heineman A, Palacio H, Bangsberg DR. Case management is associated with improved antiretroviral adherence and CD4 + cell counts in homeless and marginally housed individuals with HIV infection. Clin Infect Dis. 2006;43(2):234-42.

25. UNAIDS. Miles to go-closing gaps, breaking barriers, righting injustices. 2018. http://www.unaids.org/sites/default/files/media_asset/miles -to-go_en.pdf.

26. Koole O, Tsui S, Wabwire-Mangen F, Kwesigabo G, Menten J, Mulenga M, Auld A, Agolory S, Mukadi YD, Colebunders R, Bangsberg DR. Retention and risk factors for attrition among adults in antiretroviral treatment programmes in Tanzania, Uganda and Zambia. Tropi Med Int Health. 2014;19(12):1397-410.

27. Cornell M, Mclntyre J, Myer L. Men and antiretroviral therapy in Africa: our blind spot. Trop Med Int Health. 2011;16(7):828-9.

28. Messeri PA, Abramson DM, Aidala AA, Lee F, Lee G. The impact of ancillary HIV services on engagement in medical care in New York City. AIDS Care. 2002;14(Suppl 1):S15-29.

29. Rothman J, Rudnick D, Slifer M, Agins B, Heiner K, Birkhead G. Co-located substance use treatment and HIV prevention and primary care services, New York State, 1990-2002: a model for effective service delivery to a high-risk population. J Urban Health. 2007:84(2):226-42.

30. French T, Tesoriero J, Agins B. Changes in stress, substance use and medication beliefs are associated with changes in adherence to HIV antiretroviral therapy. AIDS Behav. 2011;15(7):1416-28.

31. French T, Weiss L, Waters M, Tesoriero J, Finkelstein R, Agins B. Correlation of a brief perceived stress measure with nonadherence to antiretroviral therapy over time. J Acquir Immune Defic Syndr. 2005;38(5):590-7.

32. Sitapati AM, Limneos J, Bonet-Vazquez M, Mar-Tang M, Qin H, Mathews WC. Retention: building a patient-centered medical home in HIV primary care through PUFF (Patients Unable to Follow-up Found). J Health Care Poor Underserved. 2012;23(3 Suppl):81-95.

33. Gross R, Bellamy SL, Chapman J, Han X, O'Duor J, Palmer SC, et al. Managed problem solving for antiretroviral therapy adherence: a randomized trial. JAMA Intern Med. 2013;173(4):300-6.

34. USAID. CLIMATE RISK SCREENING FOR FOOD SECURITY KARAMOJA REGION, UGANDA. 2017. https://www.usaid.gov/sites/default/files/ documents/1866/170130_Karamoja_Food_Security_Climate_Screening. pdf.

35. Geng EH, Nash D, Kambugu A, Zhang Y, Braitstein P, Christopoulos KA, et al. Retention in care among HIV-infected patients in resource-limited settings: emerging insights and new directions. Curr HIV/AIDS Rep. 2010;7(4):234-44.

36. Geng EH, Bangsberg DR, Musinguzi N, Emenyonu N, Bwana MB, Yiannoutsos CT, et al. Understanding reasons for and outcomes of patients lost to follow-up in antiretroviral therapy programs in Africa through a sampling-based approach. J Acquir Immune Defic Syndr. 2010:53(3):405-11.

37. PEPFAR. Improving access to HIV treatment services through community ART distribution points in Uganda. Washington, D.C. PEPFAR; 2018. https ://www.pepfarsolutions.org/women/2018/1/13/improving-access-to-hivtreatment-services-through-community-art-distribution-points-in-ugand 